Ingrid Gogolin (2019)

\title{
Durchgängige Sprachbildung
}

\section{Kontext der Entwicklung des Konzepts}

Das Konzept der „durchgängigen Sprachbildung“ wurde im Rahmen des Modellprogramms „Förderung von Kindern und Jugendlichen mit Migrationshintergrund (FörMig)" entwickelt, das von 2004 bis 2009 von der Bund-Länder-Kommission für Bildungsplanung und Forschungsförderung (mit einer Transferphase bis 2013) gefördert wurde (Gogolin et al., 2011). Hauptaufgabe dieses Programms war die Entwicklung und praktische Erprobung von Konzepten der Förderung, die geeignet sein sollten, Brüche in der Bildungslaufbahn von Kindern und Jugendlichen zu vermeiden. Solche Brüche sind angesichts der Struktur des deutschen Bildungssystems an mehreren Stellen der Bildungsbiographie zu erwarten: beim Übergang von der vorschulischen Einrichtung in die Grundschule, von der Grundschule in eine der Formen der Sekundarschule, von Schulformen der Sekundarstufe I in eine berufliche oder weiterführende allgemeine Bildung. So findet anders, als in den meisten Bildungssystemen der Welt, im größten Teil der deutschen Bundesländer der Übergang vom Primar- in den Sekundarbereich bereits nach vier Jahren Grundschule statt. Das Konzept der „durchgängigen Sprachbildung” ist deshalb in erster Linie darauf gerichtet, dass die (im internationalen Vergleich zahlreichen) Übergänge, mit denen auch ein Wechsel der Institution verbunden ist, nicht als Bruch in der (Sprach-) Bildungsbiographie erfahren werden, sondern als aneinander anschließend und weiterführend. Grundlage für die Entwicklung des Konzepts war eine umfassende Bestandsaufnahme des Forschungsstands zur Sprachentwicklung und Sprachbildung im Kontext von Mehrsprachigkeit.

\section{Dimensionen durchgängiger Sprachbildung}

„Durchgängige Sprachbildung“ in diesem Verständnis besitzt drei Dimensionen. Die erste, die sog. bildungsbiographische Dimension, weist darauf, dass Sprachbildung über die gesamte Bildungsbiographie hinweg erforderlich ist. Die zweite, die sog. Kooperationsdimension betrifft das Erfordernis, dass eine Zusammenarbeit aller am Sprachbildungsprozess beteiligten Partner(innen) ratsam ist. Die dritte, die sog. Mehrsprachigkeitsdimension betrifft die Beziehungen und Verbindungen zwischen den verschiedenen Sprachen oder Varietäten, in denen eine Person lebt und die sie lernt. 


\section{Bildungsbiographische Dimension}

Die Forschung zu Spracherwerb bzw. Sprachentwicklung im Verlauf von Bildungsbiographien zeigt an, dass sich über den gesamten Bildungsprozess hinweg die Aufgabe der Sprachbildung stellt. Ein wichtiger Grund dafür ist, dass der Ausbau der sprachlichen Mittel an konkrete Spracherfahrungen gebunden ist. In der Phase des allerersten Spracherwerbs eignen sich Kinder auf intuitive Weise die Fundamente für ihre sprachlichen Fähigkeiten an. Hierfür verantwortlich ist das Zusammenspiel von angeborenen Dispositionen zum Spracherwerb mit dem sprachlichen Input, den sie in der Kommunikation mit ihrer unmittelbaren Umgebung erfahren. Dabei kann es sich um eine Sprache handeln, aber durchaus auch um zwei oder mehr Sprachen. In diesem Prozess bauen Kinder den Grundstock an Strukturmitteln auf (also z. B. das grammatische Grundgerüst), das ihrer oder ihren Umgebungssprache(n) eigen ist.

Je weiter der Spracherwerb fortschreitet, desto mehr ist die Entwicklung abhängig vom konkreten Erleben sprachlicher Mittel (Weinert, 2013). Die intuitive Aneignungsweise, die die große Stärke beim ersten Spracherwerb ausmacht, tritt allmählich hinter eher kognitive Strategien zurück - Strategien also, die mit Wissen über Sprache und ihre Funktionsweisen verbunden sind (List, 2011). Spätestens mit dem Eintritt in die Schule wird der Sprachausbau stark von kognitiven Prozessen beeinflusst, etwa beim Lesen- und Schreibenlernen, in dem ein Kind begreifen muss, dass es hier vor allem darum geht, dass Lautzeichen und Schriftzeichen lediglich durch die darüber historisch entstandenen Konventionen miteinander verbunden sind, nicht aber durch ein quasi-natürliches Verhältnis (wie etwa, dass man bei "genauem Zuhören" hören könne, wie etwas geschrieben wird. Tatsächlich kann man Schreibzeichen nur "hören", wenn man genau weiß, wie sie geschrieben werden).

Erfolgreiche Sprachaneignung zeichnet sich insgesamt dadurch aus, dass die Lernenden von ihrer alltäglichen Sprachpraxis abstrahieren und differenzieren lernen. Im Verlaufe der Bildungsbiographie werden die sprachlichen Mittel, die eine lernende Person verstehen und selbst beherrschen muss, immer spezifischer - abhängig davon, wie sich die Themen und Gegenstände (z.B. in die Fächer der Schule oder tertiärer Bildungseinrichtungen) ausdifferenzieren. Die sprachlichen Mittel beispielsweise, die für die Beschäftigung mit dem Thema „Kraft” im grundschulischen Sachunterricht verwendet werden, unterscheiden sich von den Mitteln, die für die Behandlung desselben Themas im Physikunterricht der Sekundarstufe erforderlich sind (vgl. z. B. Tajmel, 2013). Diese Unterscheidungen sind nicht nur auf der Ebene der Wörter relevant (also für den „Fachwortschatz"), sondern auch auf der Textebene: Ein Text über „Kraft” in der Grundschule wird mit konkretisierenden Illustrationen arbeiten („Der Wind hat viel Kraft, er kann den Baum verbiegen..."), während im Text für die Sekundarstufe eher abstrakte Gesetzmäßigkeiten in den ihnen eigenen, verdichteten Ausdrucksweisen formuliert sind („Physikalische Kraft kann den Bewegungszustand eines Körpers verändern..."). Die bildungsbiographische Dimension der "durchgängigen Sprachbildung" zeigt also an, dass die Rede- 
mittel, die notwendig sind, um die im Bildungsprozess sich wandelnden sprachlichen Anforderungen zu erfüllen, jeweils zu dem Zeitpunkt und in dem Kontext vermittelt werden müssen, zu dem sie der Sache nach erforderlich sind.

\section{Kooperation}

Die zweite Dimension der "durchgängigen Sprachbildung" betrifft das Problem der Effizienz von Sprachbildungsmaßnahmen. Im Verlaufe einer Bildungsbiographie haben Lernende eine zunehmende Fülle und Spezifik an sprachlichen Mitteln zu bewältigen. Diese Mittel sind zu erheblichen Teilen an den Kontext des Lernens in einer formalen Bildungseinrichtung gebunden. Das bedeutet einerseits, dass sie zu weiten Teilen auf den Gesetzmäßigkeiten konzeptioneller Schriftlichkeit beruhen (also der „Bildungssprache“ zuzurechnen sind; vgl. hierzu Gogolin, 2010; Gantefort, 2013). Andererseits sind sie, wie bereits ausgeführt, zunehmend gegenstands- oder fachspezifisch. Hieraus folgt zum einen, dass der Ausbau der entsprechenden Fähigkeiten sich im Alltagssprachgebrauch kaum vollzieht, da dieser überwiegend im Modus konzeptioneller Mündlichkeit vonstattengeht. Es sind vielmehr systematische Anleitungsprozesse durch die Bildungsinstitutionen erforderlich. Zum anderen folgt daraus, dass eine arbeitsteilige Vorgehensweise zwischen den am Sprachbildungsprozess Beteiligten ratsam ist. Im einzelnen Lehr-/ Lernarrangement (z. B. im Unterrichtsfach) sollte der Fokus auf den dafür spezifischen sprachlichen Mitteln liegen. Aber es bedarf zugleich der Abstimmung zwischen den Lehr-/ Lernbereichen, damit nicht einerseits längst Gelerntes überflüssigerweise wieder und wieder angeboten wird, oder andererseits benötigte sprachliche Mittel ausgespart bleiben, weil niemand die Zuständigkeit dafür übernommen hat. Auch zwischen Instanzen und Personen, die das Lernen im nonformalen und informellen Sektor unterstützen, und den formalen Einrichtungen (wie Kindertagesstätte oder Schule) sind Abstimmungen erforderlich, damit es nicht ungewollt zu widersprüchlichen, einander eher konterkarierenden als unterstützenden Angeboten kommt. Die Koordination der Elemente sprachlicher Bildung und die Kooperation der Beteiligten sind also erforderlich, damit die Lernenden in der insgesamt geringen zur Verfügung stehenden Lernzeit optimale Lernmöglichkeiten erhalten.

\section{Mehrsprachigkeit}

Mit dem Hinweis auf Mehrsprachigkeit im Konzept der „durchgängigen Sprachbildung" wird darauf aufmerksam gemacht, dass jedes bereits erworbene sprachliche Können und Wissen eine Basis dafür ist, die nächste Hürde zu nehmen. Angeknüpft wird hierbei an zwei theoretischen Grundlagen: Aus entwicklungstheoretischer Sicht ist es für erfolgreiches Lernen bedeutsam, dass das Angebot, das der lernenden Person gemacht wird, an dem momentan vorhandenen Kenntnis- und Entwicklungsstand anknüpft („Zone der nächsten Entwicklung“, vgl. Vygotskij, 1934/2002). Unterstützt durch Lehrende (im weiteren Sinne) wird auf dieser Grundlage der nächste Schritt der Aneignung von Kenntnissen oder Fähigkeiten vollzogen. Bedeutend dabei ist, dass jede sprachliche Vorerfahrung bedeutsam für den nächsten An- 
eignungsschritt ist. Im Anschluss an Wandruszka (1981) entwickelt jedes Individuum Formen der Mehrsprachigkeit: Im Lebensverlauf werden zahlreiche Varianten einer Sprache erworben (soziale Varianten, Dialekt- und Standardvarianten oder auch die Varianten, die von Expert(inn)en für ein Thema oder Gebiet benutzt werden). Zusätzlich zu dieser „innersprachlichen Mehrsprachigkeit” werden Erfahrungen mit sprachenübergreifenden Formen der Mehrsprachigkeit gewonnen. Nahezu 100 Prozent der Kinder, die in Deutschland eine Schule besuchen, lernen eine Fremdsprache (zumeist Englisch). Für einen erheblichen Teil der Schülerschaft nämlich alle jene, die einen höheren Bildungsabschluss anstreben - ist eine zweite Fremdsprache obligatorisch. Hinzu kommt ein ebenfalls erheblicher Teil der Schülerschaft mit lebensweltlichen Erfahrungen in mehr als einer Sprache; dies sind alle diejenigen, die in Familien aufwachsen, in denen eine oder mehrere andere Sprache(n) neben Deutsch gebraucht werden. Diese - unterschiedlichen - Spracherfahrungen bilden die Ressourcen, an die sprachliche Bildung anknüpfen muss.

\section{Fazit}

Eine bedeutende Grundlage der "durchgängigen Sprachbildung" ist es, dass die angeleiteten Lernprozesse immer wieder auf der Basis von Vergewisserung über das bereits Erreichte gestaltet werden. Aufgegriffen werden hier Methoden der prozessbegleitenden Sprachdiagnostik, also solche, die eine Dauerbeobachtung der Entwicklung von Lernenden erlauben. Anregungen dafür bieten Handreichungen wie die "Niveaubeschreibungen Deutsch als Zweitsprache" (Sächsisches Bildungsinstitut 2013). Sie sind besonders auf die Anforderungen der Bildungspraxis zugeschnitten, und die mit der Unterstützung dieser Instrumente festgehaltenen Beobachtungen der sprachlichen Entwicklung von Lernenden ergeben eine gute Basis für die Gestaltung „durchgängiger Sprachbildung”.

\section{Literatur}

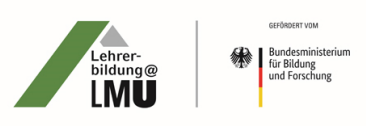

Gantefort, Ch. (2013). Bildungssprache. Merkmale und Fähigkeiten im sprachtheoretischen Kontext. In I. Gogolin, I. Lange, U. Michel \& H.H. Reich (Hrsg.), Herausforderung Bildungssprache - und wie man sie meistert (S. 71-105). Münster u.a.: Waxmann (FÖRMIG-Edition, 9).

Gogolin, I., Dirim, İ., Neumann, U., Reich, H. H., et al. (2011). Förderung von Kindern und Jugendlichen mit Migrationshintergrund. Bilanz und Perspektiven eines Modellprogramms (FörMig Edition). Münster \& New York: Waxmann.

Gogolin, I. (2010). Was ist Bildungssprache? Grundschule Deutsch (4), S. 4-5. List, G. (2011). Spracherwerb und die Ausbildung kognitiver und sozialer Kompetenzen. Deutsches Jugendinstitut. München (WIFF Expertise). https://www.weiterbildungsinitiative.de/uploads/media/WiFF Expertise 11 List I nternet.pdf [Zugriff 20.4.2019]

Sächsisches Bildungsinstitut (Hrsg.) (2013). Niveaubeschreibungen Deutsch als Zweitsprache (Primarstufe; Sekundarstufe I und II). Radebeul (Landesamt für Schule und Bildung). 
Tajmel, T. (2013). Bildungssprache im Fach Physik. In I. Gogolin, U. Michel \& H.H. Reich (Hrsg.). Herausforderung Bildungssprache. FörMig-Edition (S. 239-256). Münster \& New York: Waxmann.

Vygotskij, L. S. (1934/2002). Denken und Sprechen. Herausgegeben und aus dem Russischen übersetzt von Joachim Lompscher und Georg Rückriem. Weinheim $\&$ Basel: Beltz.

Wandruszka, M. (1981). Die Mehrsprachigkeit des Menschen. München: Deutscher Taschenbuch-Verlag.

Weinert, S., Ebert, S. (2013). Spracherwerb im Vorschulalter. Soziale Disparitäten und Einflussvariablen auf den Grammatikerwerb. Zeitschrift für Erziehungswissenschaft (ZfE) 16 (2), S. 303-332. 